\title{
Modeling of Force Feedback and Research on Control Strategy of Returnability of Steering Wheel
}

\author{
Zhe Wang ${ }^{1, a}$, Qian $\mathrm{Ye}^{2, \mathrm{~b}}, \mathrm{Na} \mathrm{Hu}^{3, \mathrm{c}}$, Mangmang Hang ${ }^{4, \mathrm{~d}}$ \\ ${ }^{1}$ School of Mechanical Engineering, ZhejiangUniversity, Hangzhou, china \\ ${ }^{2}$ Zhejiang Academy of Building Research \& DesignCO.LTD, Hangzhou, china. \\ ${ }^{3}$ School of Mechanical and ElectronicEngineering ,WuhanUniversity of Technology, Wuhan, china. \\ ${ }^{4}$ School of Mechanical Engineering, ZhejiangUniversity, Hangzhou, china \\ aEmail:wzhe08@163.com, ${ }^{\mathrm{b}}$ Email:melty0827@126.com, ${ }^{\mathrm{c} E m a i l}$ :hqwdyd@163.com
}

Keywords:steering wheel; force feedback; control strategy;

\begin{abstract}
A force feedback model of steering wheel was established based on mechanical analysis of the vehicle steering system. Taking driver's preference for steering torque into consideration, a correction coefficient was introduced to this model, and a method for determining the coefficient was also put forward. To study on the steering wheel return control strategy, the relationship between torque and speed as well as voltage of the torque motor was analyzed by interpolation fitting. In addition, the simulated control model in Matlab/Simulink was set up. As for the oscillation problem, in order to improve the steering wheel returnability, Improvement of control strategy of torque motor was proposed. The model was further optimized through the research of the influence of different elastic coefficient and damping coefficient on steering wheel returnability. Finally, the real experiment proved the feasibility of the model and control strategy.
\end{abstract}

\section{Introduction}

The force feedback characteristics of steering wheel refer to the law of the steering wheel torque feedback varying with the vehicle motion state. Road condition, driver's operation behavior and other factors will affect force feedback of the steering wheel. That the vehicle steering system has good feedback force characteristics is premise for driver to better control of the vehicle .In previous researches, Fuzzy control was applied to road feelcontrol in steer-by-wire system[1] . Additionally, researcher analyzed automobile force by Calman filter and designed the road-feel simulation method of which was closing to the electric power steering system[2] .But these methods are relatively complex and difficult operation, This paper attempts to study a simple, practical method of building feedback model and discussing returnability control strategy

\section{Steering wheel force feedback model}

Steering force torque feedback of steering wheel can be regarded as resistance torque transmitted from steered wheel to steeringwheel, this steering resistance torque includes the friction resistance torque caused by contacting with the road and aligning torque of the steering wheel. When steering, aligning torque relevant to front wheel lateral forcecan not be too small in principle and the friction which has nothing to do with the front wheel lateral force should be as small as possible [3].In this paper, the main consideration is the aligning torque of steering wheel caused by steered wheel returning. The steered wheel aligning resistance torque includes two parts: one part is caused by the tire drag which consists of air drag and tilt drag, this torque is proportional to the centrifugal acceleration; The other part is caused by kingpin inclination and displacement, this torque has nothing to do with speed, it can be calculated according to the vehicle parameters.

Aligning torque caused by tire drag can be regarded as the product of the lateral force $F_{y}$ and the tire drag after mechanical property of vehicle steering analyzed. 


$$
M_{Z V}=F_{y} \cdot\left(\varepsilon_{1}+\varepsilon_{2}\right) \cdot(1)
$$

Where, $M_{Z V}$ is the aligning torque caused by the tire drag, $\varepsilon$ is the tire drag, $\varepsilon_{1}$ is tilt drag, $\varepsilon_{2}$ is air drag.

The equation of aligning torque $M_{A}$ caused by kingpin inclination and displacement is:

$$
M_{A}=\frac{Q_{w} S}{2} \sin (2 \gamma) \sin \theta \cdot(2)
$$

Where, $\mathrm{S}$ is the kingpin shift, $Q_{w}$ is the wheel load, $\gamma$ is the kingpin inclination, $\theta$ is the front wheel angle. Considering moment of inertia and damping of the vehicle steering system, the steering wheel aligning torque can be expressed as follows:

$$
M_{w}=\frac{M_{Z V}+M_{A}}{i}+C_{s} \dot{\theta}_{w}+J_{s} \ddot{\theta}_{w} \cdot(3)
$$

Where, $M_{w}$ is the steering wheel aligning torque, $i$ is the drive ratio, $C_{s}$ is the damping coefficient of steering column, $\theta_{w}$ is the steering wheel, $J_{s}$ isthe steering wheel moment of inertia.

The lateral force is the main reason for the deformation of the steering wheel, when vehicle driving, the sum of all tire lateral force are approximately proportional to the centripetal acceleration [5], i.e.

$$
F_{y}=m \frac{v^{2} l_{b}}{R l} .(4)
$$

Where, $m$ is the mass of vehicle, Ris turning radius, $v$ is speed, $l$ is the wheelbase, $l_{a}$ is the distance from centroid to the front wheel shaft and $l_{b}$ is from centroid to the rear wheel shaft, $k_{1}, k_{2}$ are respectively the frontand rear wheel cornering stiffness. Turning radius calculation formula:

$$
\mathrm{R}=\left(l+m \frac{k_{2} l_{b}-k_{1} l_{a}}{k_{1} k_{2} l}\right) / \theta .
$$

The relationship between the front wheel angleand steering wheel angle can be expressed by the following equation:

$$
\theta=\frac{\theta_{w}}{i}(6)
$$

Usually $i$ is large, $\theta$ is small, so it can be Simplified as $\sin \theta \approx \frac{\theta_{w}}{i}$. For easily representation, two parameters are introduced.

$$
\begin{aligned}
& K^{\prime}=\left[\left(m v^{2} l_{b}\right) /\left(l^{2}+m \frac{k_{2} l_{b}-k_{1} l_{a}}{k_{1} k_{2}} v^{2}\right)\right]\left(\varepsilon_{1}+\varepsilon_{2}\right), \\
& K^{\prime \prime}=\frac{Q_{w} s}{2} \sin (2 \gamma) .
\end{aligned}
$$

In addition, in the normal driving, driver operate slowly the steering wheel, so its angle acceleration is small, in order to simplify the model, angle acceleration can be ignored . Then $M_{w}$ can be expressed as:

$$
M_{w}=\frac{K^{\prime}+K^{\prime \prime}}{i^{2}} \theta_{w}+C_{s} \dot{\theta}_{w}(7)
$$


Main model parameters of a vehicle are as followed: $m=1760 \mathrm{~kg}, l=2.774 \mathrm{~m}, \varepsilon_{1}+\varepsilon_{2}=$ $0.07 \mathrm{~m}, l_{a}=1.04 \mathrm{~m}, l_{b}=1.56 \mathrm{~m}, k_{1}=k_{2}=35000 \mathrm{~N} / \mathrm{rad}, \mathrm{S}=0.2 \mathrm{~m} \mathrm{Q} \mathrm{Q}_{\mathrm{w}}=5000 \mathrm{~N}, \gamma=$ $0.14 \mathrm{rad}, i=15, J_{s}=0.01 \mathrm{Kg} / \mathrm{m}^{2}, C_{s}=0.1 \mathrm{~N} \cdot \mathrm{m} \cdot \mathrm{s} / \mathrm{rad}$.(7) is obtained based on the analysis of the theory of traditional steering systemwithout considering the influence of power assist system, for example, when $\mathrm{v}=30 \mathrm{~km} / \mathrm{h}, \theta_{w}=\pi / 3$, according to (7) there is $M_{w}=25.88 \mathrm{~N} \cdot \mathrm{m}$, which is significantly exceed driver'spreference for the steering wheel torque obtained by General Companytest described in[6] ,So a correctioncoefficientis introduced, (7) can be modified:

$$
M_{w}=C_{s} \dot{\theta}_{w}+\frac{K^{\prime}+K^{\prime \prime}}{\lambda i^{2}} \theta_{w}(8)
$$

Establish the virtual prototype model in ADAMS, because steering wheel speed is small inthesimulation experiment, and related coefficient of speed is small too, so the relationship between the steering torque and steering speed is neglected here. Through simulation we can get thesteering torque-angle curve of the corrected system underdifferent speeds of $30,40,50 \ldots 100 \mathrm{~km} / \mathrm{h}$.

By fitting thesimulation curve and compared with (8), the corresponding correction coefficients areattained respectively as shown inFig.1. With comprehensive consideration, take $\lambda=6$.
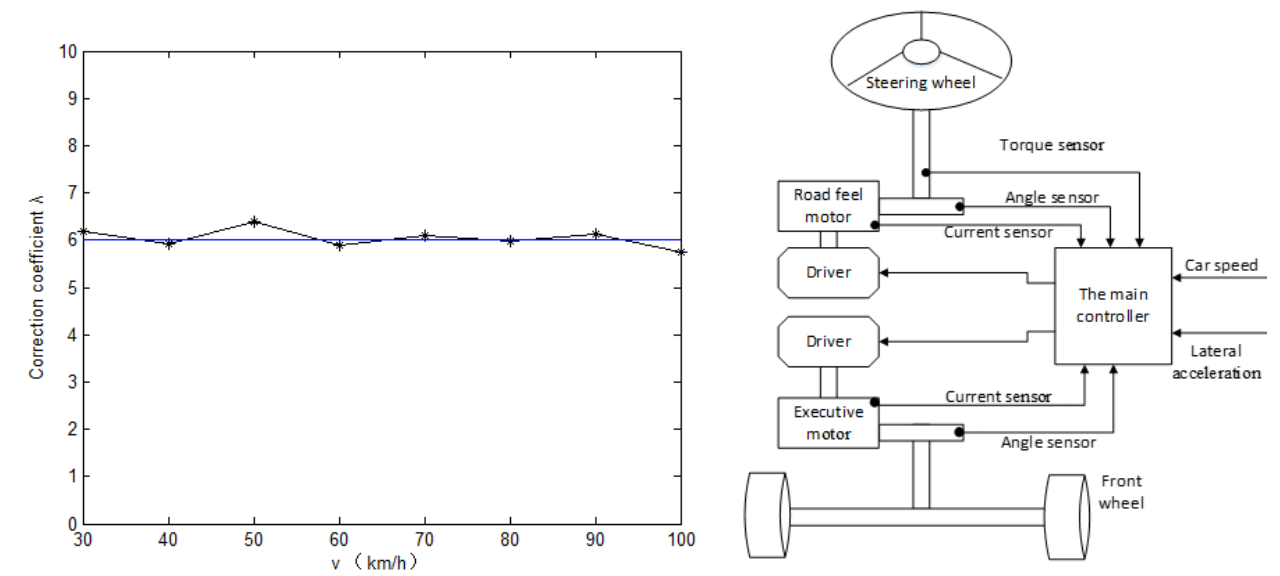

Fig.1. Correction coefficients of different speeds Fig.2. Steer-by-wire system

\section{The study of control strategy of steering wheel returnability}

Mechanical connectionbetween the steered wheel and the steering wheel has been cancelled in the steer-by-wire simulation system ,Instead the aligning torqueis simulated by controlling the motor torque, and express real-time road feelto the driver, so that the driver can maser the vehicle running state and road condition, the corresponding way is as shown in Fig.2. In the case of constant speed of the vehicle and slow rotation of the steering wheel, the simplest effect of the steering wheel is linear feedback, driver operating the steering wheel can obtain sensation like spring force.

The experiment motor adopts 5TK20GN-CW2E AC torque motormade in Japan OrientalMotor Company. Output torque of the motor is related with set voltage $\mu$ and motor speed $n$. As the set voltage is discrete, which cannot exactly express motor characteristics under any set of voltage. So the relationship among torque, voltage and speed is fitted with interpolation algorithm according to characteristic curve of the motor itself.

$$
\mathrm{T}=\left\{\begin{array}{c}
(4-\mu) T_{3}(n)+(\mu-3) T_{4}(n),(0 \leq \mu \leq 4) \\
\left(\mu^{2}-10 \mu+25\right) T_{4}(n)+\left(-\mu^{2}+10 \mu-24\right) T_{5}(n),(4<\mu \leq 5)
\end{array}\right.
$$

Where, $T_{3}(n), T_{4}(n), T_{5}(n)$ are functions of the relationship between speed and torque, which is the inherent characteristic of the torque motor.

Keep constant speed, Turn the steering wheel to a certain angle, then loosen the steering wheel the steering wheel will return back on the drive of aligning torque, which is called returnability of the 
steering wheel. Built corresponding control model in Simulink referring to the established force feedback model. Then the return torque can be controlled by controlling the torque motor [4].In simulation experiment, set the initial steering wheel angle for 1.5rad, and then release the steering wheel, in the active returnprocess, angle displacement curve of the steering wheel is shown in Fig.3.

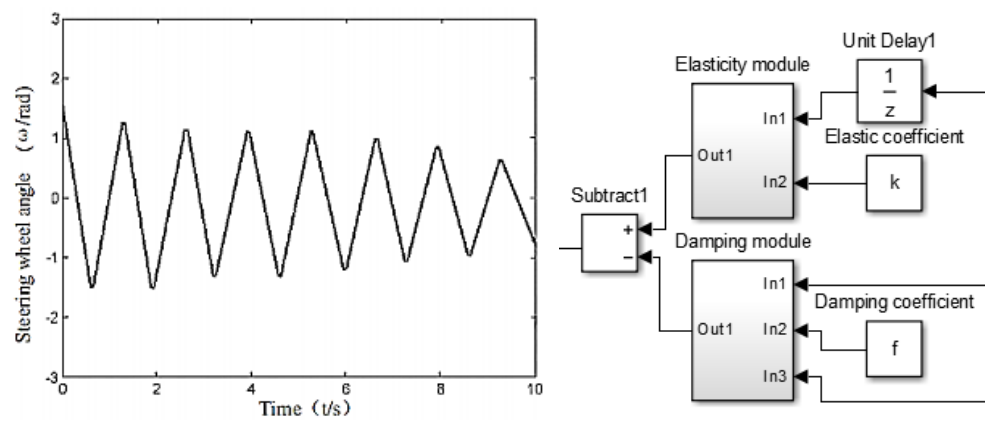

Fig.3. Angle displacement curve

Fig.4. Damping module and elastic of the steering wheelmodule in Simulink

It can be seen from Fig.3 that the steering wheel shocksin active return process, the real experiment results are consistent with the simulation's, In order to enhance the steering wheel returnability and make sure the steering wheel returning stably and quickly, the control strategy of the motor should be improved. One feasible way is to add a damping module in the control module of the motor. Since the elastic module and the damping module output a control voltage together after added, so the torque $M_{w}$ concerned withsteering wheel angle also need to adjust [7].

$$
M_{w}=\left(f+C_{s}\right) \dot{\theta}_{w}+k\left(\frac{K^{\prime}+K^{\prime \prime}}{\lambda i^{2}}\right) \theta_{w}
$$

Where, $k$ is the elastic coefficient, $f$ is the damping coefficient. the modules added in Simulink ara as shown in Fig.4.

Setting differentcoefficients of elasticity and damping, Different return curve of steering wheel are as shown in Fig.5 .By comparison, we can see, the steering wheel returns the fastest and its oscillation is minimum when $f=0.4$.
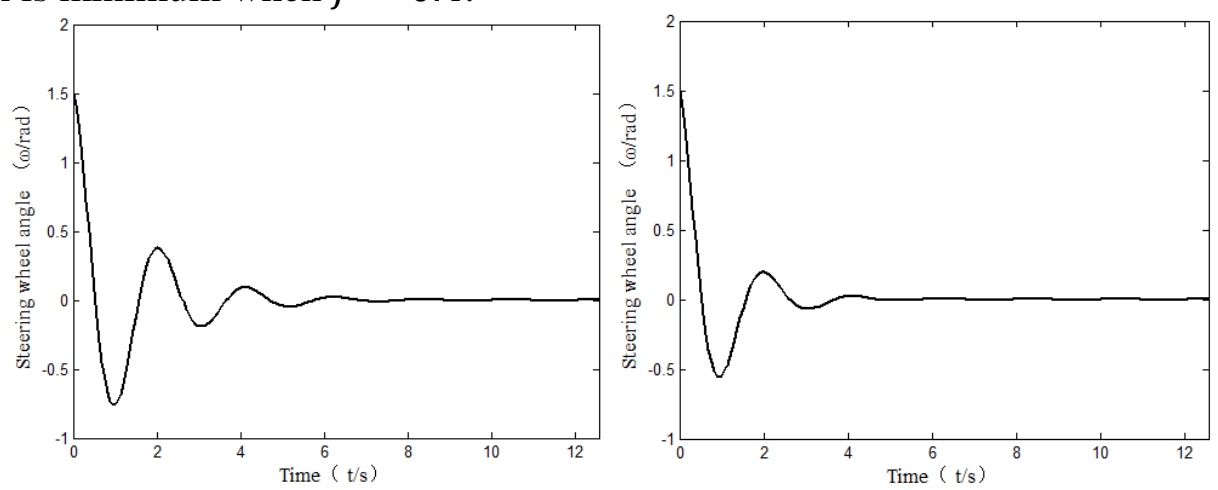

(a) $f=0.1 k=0.6$ (b) $f=0.2 k=0.6$ 

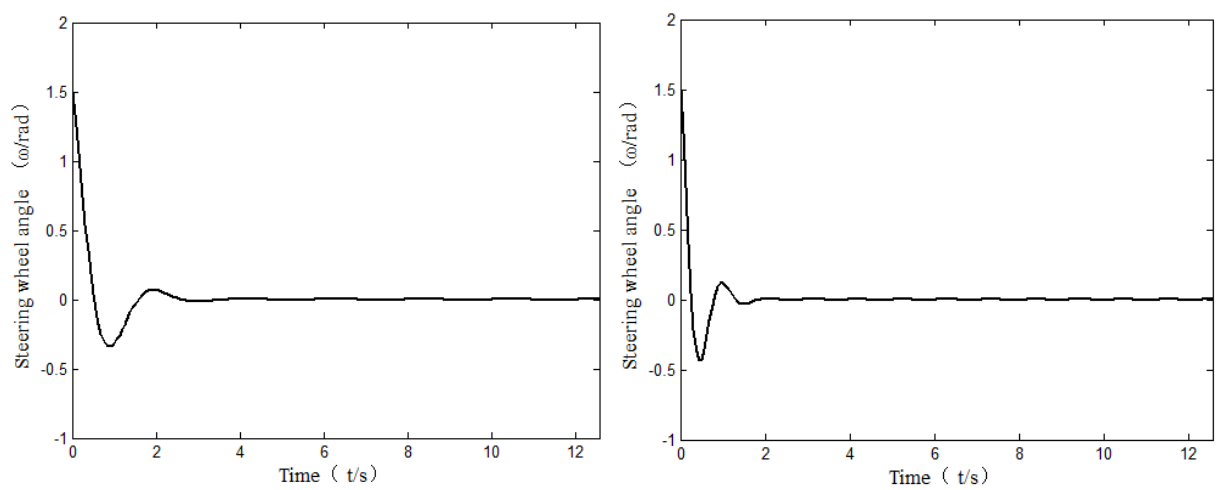

(c) $f=0.3 k=0.6$ (d) $f=0.4 k=0.6$

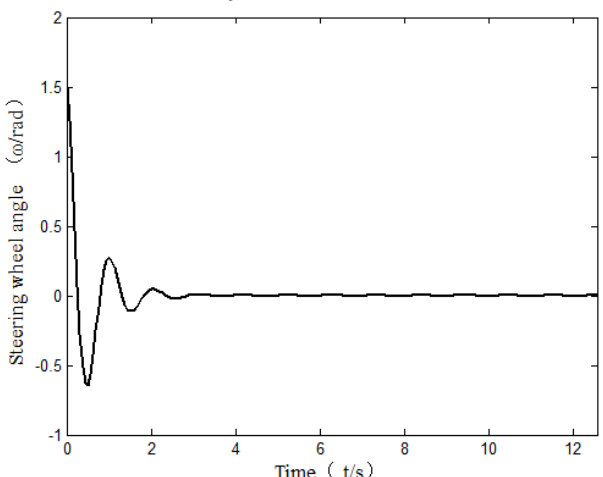

(e) $f=0.5 \quad k=0.6$

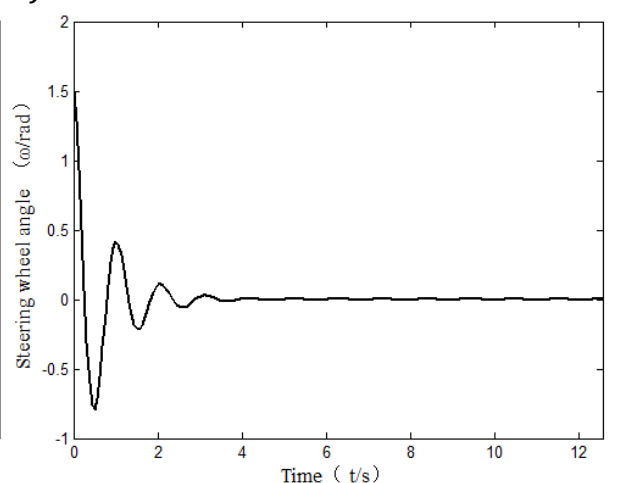

(f) $f=0.6 \quad k=0.6$

Fig.5. Return curve of steering wheel

Similarly, set $f=0.4$, change $k$, different steering wheel return curve can be gotten. However, it canbe found that $k$ has little effect on thereturn time, but has great influence on the oscillation amplitude of the steering wheel. The smaller $k$ is, the weaker the oscillation will be. But k cannot be too small in view of the fact that elastic coefficient have much to do with the steering wheel aligning torque which can affect driver's road feel. Withcomprehensive consideration, $\mathrm{k}=0.6$.In summary, when $f=0.4, k=0.6$, the returnability of steering wheel is best.

\section{Test results}

Test bench of the steering simulation system is shown in Figure 6. Test the result parametersacquired from the Simulation on the bench, the specific test method refer to the national standard GB / T6323. 4-1994.

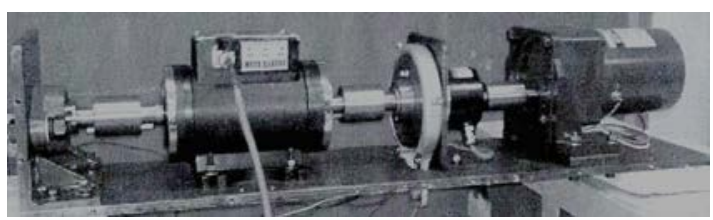

Fig.6. Test bench of the steering simulation system

When the vehicle speed is stable, spinning slowly the steering wheel, it shows that steering wheel torque and angle substantially exhibits a linear relationship.At this time, the damping module does not play a role, which is consistent with the theoretical analysis.

Set $\mathrm{k}=0.6$, $\mathrm{f}=0.4$, keep vehicle speed $30 \mathrm{~km} / \mathrm{h}$ constantly,Turn the steering wheel to a certain angle, Thentake away hands and let it go, Results are showed in Fig.7and Fig.8.From these two curve, we can see whether it is positive or reverse, the steering wheel can return back quickly, the oscillation amplitude is also very small, which refers good returnability of the steeringwheel. 

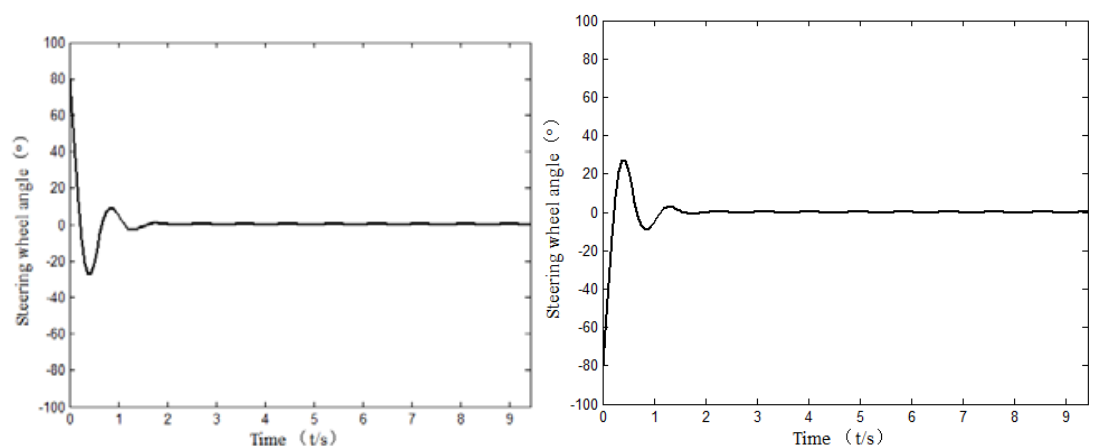

Fig.7. Positive return curve of steering wheel Fig.8. Reverse return curve of steering wheel

\section{Conclusions}

(1) The basic model of steering wheel force feedback was built. The correction coefficient was acquired with the help of ADAMS simulation. Then the relationship between torque and voltage, speed of the experimental torque motor was obtainedthrough interpolation fitting. Finally, the oscillation problem affecting returnability was conquered by analyzing different elastic coefficient and damping coefficient in Matlab/Simulink, The simulation results show that, when $f=0.4, k=$ 0.6, the steering wheel can basically eliminate the oscillation and return as soon as possiblewhich was in accordance with the real experiment results, thus verifying the correctness of the model and the control strategy.

(2) The process of modeling of force feedback in this paper is simple, the method of model updating and optimization has a Strong operability, so the research provides a practical reference to products development of automobile driving simulator.

\section{References}

[1] Wu Jin, TianJie, Luo Shi. Fuzzy PID Based Controller of Road Feeling for Steer-by-wire System [J] .Mechanical Science and Technology for Aerospace Engineering.2012, 31(6):919-923.

[2] Hong yu Zheng, Chang fuZong, Xiang Wang. Road Feel Design for Vehicle Steer-by-wire System [J]. Journal of agricultural machinery, 2011, 42(2):18-22.

[3] Mickey M. vehicle dynamics C volume (Second Edition) [M]. Beijing: people traffic Press,1997.

[4] O denthalD, Bunte T, Heitzer HD.et a.l How toMake Steer-by-wire Feel like Power Steering[C]. 15th IFAC World Congress on Automatic Control, Barcelona, 2002.

[5] Gillespie T. D ,liuqi zhao,dafeng Jin. Fundamentals of vehicle dynamics [M]. Beijing: Tsinghua University press, 2006.

[6] BertolliniGP, HoganRM .Applying Driving Simulation to Quantify Steering Effort preference as a Function of Vehicle Speed[C].SAE Paper.1999.

[7] Mangmang Huang.Research and Development of Steering Simulation System in Vehicle Driving Simulator[D], Zhejiang University,2012.3.

[8] Shengbing Yang. Steer-by-wire System Control Strategy Research [D], Wuhan University of Technology, 2008.5. 